\title{
ON THE FUNDAMENTAL GROUPS OF NEGATIVELY CURVED MANIFOLDS WITH FINITE VOLUME
}

\author{
MIDORI S. GOTO ${ }^{1}$
}

\begin{abstract}
We will prove that if $M$ is a complete, simply connected riemannian manifold with sectional curvature $K, a<K<0$, for $a>0$ and $\Gamma$ a properly discontinuous group of isometries of $M$ acting freely on $M$ with volume $(M / \Gamma)$ finite, then $M / \Gamma$ is compact if and only if $\Gamma$ consists of hyperbolic elements.
\end{abstract}

1. Introduction. The purpose of this short note is to prove the following:

THEOREM. Let $M$ be a complete, simply connected riemannian manifold with sectional curvature $K,-a \leqslant K<0$, where $a>0$. Let $\Gamma$ be a properly discontinuous group of isometries acting freely on $M$. Suppose that the factor space $M / \Gamma$ has finite volume. Then $M / \Gamma$ is compact if and only if $\Gamma$ consists of hyperbolic elements.

This is an extension of a theorem in Heintze [6], where he assumed, moreover, $K \leqslant-b, b>0$. Our proof is straightforward using results in Gromov [4] and Heintze [6], and it seems that this is essentially much simpler and clearer than that of Heintze. For the sake of convenience, we shall add proofs for some of the known results.

2. Notation and terminologies. Throughout the paper, $M$ will denote a complete, simply connected riemannian manifold of dimension $n$, with sectional curvature $K, K \leqslant 0$, and with the distance function $d$; the group of all isometries of $M$ will be denoted by $I(M)$; for an isometry $\varphi$ of $M$, we denote by $d_{\varphi}$ the corresponding displacement function of $\varphi: d_{\varphi}(p)=$ $d(p, \varphi(p))$ for $p \in M$; for a subgroup $\Gamma$ of $I(M)$ we adopt the notation

$$
d_{\Gamma}(p)=\min _{1 \neq \varphi \in \Gamma} d_{\varphi}(p) \quad(p \in M) .
$$

After this, in this section we suppose that $K<0$. The function $d_{\phi}$ is known to be strictly convex. An isometry $\varphi$ is said to be elliptic, hyperbolic (= axial) or parabolic if $d_{\varphi}$ has zero (minimum), positive minimum, or no minimum, respectively. For a hyperbolic isometry $\varphi$, there exists a unique geodesic $g$, which is invariant under $\varphi$ (cf. Eberlein-O'Neill [2] and Lawson-Yau [7]). The geodesic $g$ is said to be the axis of $\varphi$, and $\varphi$ acts on $g$ as a translation of the

Received by the editors April 21, 1978 and, in revised form, June 23, 1978.

AMS (MOS) subject classifications (1970). Primary 53C20; Secondary 20E40, 55A05.

Key words and phrases. Fundamental group, negatively curved manifold, hyperbolic isometry, Dirichlet region.

'Supported in part by NSF grant MCS77-18723. 
length $\min _{p \in M} d_{\varphi}(p)$. Let $\Gamma$ be a properly discontinuous group of isometries of $M$ without elliptic elements, and suppose that $\Gamma$ contains a hyperbolic element $\varphi$. Let $\Delta(\varphi)$ denote the subgroup of $\Gamma$ consisting of all elements leaving the axis $g$ of $\varphi$ invariant. Then $\Delta(\varphi)$ is a maximal cyclic subgroup in $\Gamma$. Furthermore, for hyperbolic $\varphi_{1}$ and $\varphi_{2}$, either $\Delta\left(\varphi_{1}\right)=\Delta\left(\varphi_{2}\right)$ or $\Delta\left(\varphi_{1}\right) \cap$ $\Delta\left(\varphi_{2}\right)=1$, and if $\Delta\left(\varphi_{1}\right) \cap \gamma \Delta\left(\varphi_{2}\right) \gamma^{-1} \neq 1$ for some $\gamma \in \Gamma$, then $\Delta\left(\varphi_{1}\right)=$ $\gamma \Delta\left(\varphi_{2}\right) \gamma^{-1}$. For $\Gamma$ a properly discontinuous group of isometries of $M$, the set

$$
\{q \in M: d(p, q) \leqslant d(q, \varphi(p)) \text { for } \varphi \neq 1 \text { in } \Gamma\}
$$

is called a Dirichlet region of $\Gamma$ centered at $p \in M$.

3. Results of Gromov and Margulis. We quote results from Gromov [4] for our later use.

A group is said to be almost nilpotent if it contains a nilpotent subgroup of finite index.

Proposition 1 (Gromov). Suppose that $K<0$. Let $\Gamma$ be an almost nilpotent subgroup of $I(M)$ without elliptic elements. If $\Gamma$ contains a hyperbolic element with the axis $g$, then $g$ is invariant under $\Gamma$ and $\Gamma$ is an infinite cyclic group (cf. [3] and [9]).

Proof. Let $N$ be a nilpotent subgroup of finite index of $\Gamma$. If $\psi \in \Gamma$, $\psi^{k} \in N$ for some integer $k$. Thus we may assume that $N$ contains a hyperbolic element $\varphi$ leaving the geodesic $g$ invariant. First we prove that $N$ is cyclic. Let $H_{1}$ be the group generated by $\varphi$, and $H_{2}$ the normalizer of $H_{1}$ in $N$. Every $\psi \in \mathrm{H}_{2}$ satisfies $\psi \varphi \psi^{-1}=\varphi^{k}$ for some integer $k$. Hence $\varphi^{k} \psi(g)=\psi(g)$, and $\psi(g)=g$ since $g$ is the only geodesic invariant under $\varphi^{k}$. Therefore $\mathrm{H}_{2}$ is cyclic. If $\mathrm{H}_{3}$ is the normalizer of $\mathrm{H}_{2}$, the same argument yields that $\mathrm{H}_{3}$ is cyclic. Since $N$ is nilpotent, this process stops after a finite number of steps, and it follows finally that $N$ is cyclic.

Let $N_{1}=\cap_{\gamma \in \Gamma} \gamma N \gamma^{-1}$. Then $N_{1}$ is a normal subgroup of $\Gamma$, and is contained in $N$ (and hence $N_{1}$ is also cyclic). Therefore, as any element $\psi$ of $\Gamma$ normalizes $N_{1}$, it follows that $\psi(g)=g$ and $\Gamma$ is cyclic. Thus the proof is completed.

Let $\Gamma$ be a subgroup of $I(M)$. For a given $\varepsilon>0$, we denote by $\Gamma_{\varepsilon}(p)$, $p \in M$, the subgroup of $\Gamma$ generated by all $\varphi \in \Gamma$ with $d_{\varphi}(p) \leqslant \varepsilon$, and $\Gamma_{\varepsilon}^{\prime}(p)$ the subgroup of $\Gamma_{\varepsilon}(p)$ consisting of elements whose derivative parallel translated back to $p$ is $\varepsilon$-close to the identity.

Proposition 2 (MARgulis). Suppose that $-a \leqslant K \leqslant 0$ for $a>0$. Let $\Gamma$ be a discrete subgroup of $I(M)$. Then there is a number $\varepsilon>0$ depending only on $a$ and $n(=\operatorname{dim} M)$ such that for any $p \in M, \Gamma_{\varepsilon}^{\prime}(p)$ is nilpotent and $\Gamma_{\varepsilon}(p)$ is almost nilpotent.

REMARK. The Margulis Lemma in Gromov [5] is more general than this. The above form of the statement was quoted from Thurston [8]. 
Proposition 3 (Gromov). Suppose that $-a \leqslant K<0$ for $a>0$. Let $\Gamma$ be $a$ properly discontinuous group of isometries of $M$ without elliptic elements. Let $\varepsilon$ be as in Proposition 2. Let $\pi: M \rightarrow M / \Gamma$ be the natural projection. For a maximal cyclic subgroup $\Delta$ of $\Gamma$ with a hyperbolic element, we put

$$
A(\Delta)=d_{\Delta}^{-1}([0, \varepsilon]) \text { and } C(\Delta)=\pi(A(\Delta)) .
$$

(1) Let $\Delta_{i}$ be a maximal cyclic subgroup of $\Gamma$ containing a hyperbolic element for $i=1,2$. Suppose that $\Delta_{1}$ and $\Delta_{2}$ are not conjugated to each other, i.e. $\Delta_{1} \cap \gamma \Delta_{2} \gamma^{-1}=1$ for all $\gamma \in \Gamma$. Then

$$
C\left(\Delta_{1}\right) \cap C\left(\Delta_{2}\right)=\varnothing \text {. }
$$

(2) Let $\Delta$ be a maximal cyclic subgroup of $\Gamma$ with a hyperbolic element such that $\min _{p \in M} d_{\Delta}(p) \leqslant \varepsilon / 2$. Then

$$
\operatorname{vol}(C(\Delta)) \geqslant c
$$

for some constant $c$ depending only on $n$ and $a$.

Proof. (1) Suppose that there is $p \in A\left(\Delta_{1}\right)$ with $\gamma(p) \in A\left(\Delta_{2}\right)$, where $\gamma \in \Gamma$. Then we can find $\varphi_{1} \in \Delta_{1}$ and $\varphi_{2} \in \Delta_{2}$ such that $d \varphi_{1}(p) \leqslant \varepsilon$ and $d \varphi_{2}(\gamma(p)) \leqslant \varepsilon$. Then $\varphi_{1}, \gamma^{-1} \varphi_{2} \gamma \in \Gamma_{\varepsilon}(p)$. On the other hand, $\Gamma_{\varepsilon}(p)$ is infinite cyclic by Propositions 1 and 2. Hence $\Delta_{1} \cap \gamma^{-1} \Delta_{2} \gamma \neq 1$, a contradiction.

(2) In a similar way as above, we have that $A(\Delta) \cap \gamma A(\Delta) \neq \varnothing$ for $\gamma \in \Gamma$ implies that $\gamma \in \Delta$. Let $\varphi$ be a generator of $\Delta$, and $g$ the axis of $\varphi$. We fix $p_{0}$ on $g$ and put $d\left(p_{0}, \varphi\left(p_{0}\right)\right)=d$. Then $d \leqslant \varepsilon / 2$ and, for any integer $j$,

$$
\min _{p \in M} d_{\varphi^{j}}(p)=d\left(p_{0}, \varphi^{j}\left(p_{0}\right)\right)=|j| d .
$$

Let $h(t)$ be a geodesic parametrized by arc length, with $h(0)=p_{0}$ and perpendicular to $g$. For an integer $j$ the function $f_{j}(t)=d\left(h(t), \varphi^{j}(h(t))\right)$ is strictly convex and attains the minimum $|j| d$ at $t=0$. Hence, in particular, $\lim _{t \rightarrow \infty} f_{j}(t)=\infty$. Let $k_{0}$ be the integer with $k_{0} d \leqslant \varepsilon<\left(k_{0}+1\right) d$, and we put $f(t)=\min \left\{f_{j}(t) ; j= \pm 1, \ldots, \pm k_{0}\right\}$. Then the function $f(t)$ is continuous, $f(0)=d$ and $\lim _{t \rightarrow \infty} f(t)=\infty$. Hence we can find $t_{0}$ with $f\left(t_{0}\right)=\varepsilon / 2$.

If we put $h\left(t_{0}\right)=q_{0}, d_{\Delta}\left(q_{0}\right)=d_{\varphi^{i}}\left(q_{0}\right)=\varepsilon / 2$ for some $i$ with $|i|<k_{0}$. Let $B=B q_{0}(\varepsilon / 4)$ be the open ball of radius $\varepsilon / 4$ about $q_{0}$. For any $q \in B$,

$$
d\left(q, \varphi^{i}(q)\right) \leqslant d\left(q, q_{0}\right)+d\left(q_{0}, \varphi^{i}\left(q_{0}\right)\right)+d\left(\varphi^{i}\left(q_{0}\right), \varphi^{i}(q)\right)<\varepsilon,
$$

and we have $B \subset A(\Delta)$. Furthermore, $B$ is contained in the Dirichlet region of $\Gamma$ centered at $q_{0}$, and hence $\operatorname{vol}(C(\Delta))$ is bigger than the volume of a ball of radius $\varepsilon / 4$ in a euclidean $n$-space. Thus the proof of (2) is completed.

\section{A proposition due to Heintze.}

Proposition 4 (HeINTZE). Let $\Gamma$ be a discrete subgroup of $I(M)$ acting freely on $M$. Suppose that $M / \Gamma$ has finite volume and is noncompact. Let $\pi$ denote the projection map $M \rightarrow M / \Gamma$. Let $g$ be a geodesic ray in $M$ such that $\pi \cdot g$ is also a minimizing geodesic ray which goes to $\infty$ in $M / \Gamma$. Then for a given $\varepsilon>0$, 
there is a positive number $t_{0}$ such that for $t \geqslant t_{0}$ there exists an isometry $\varphi$ $(\neq 1)$ in $\Gamma$ wi $\$ d d(\varphi(g(t)), g(t))<\varepsilon$.

Proof. For a given $\varepsilon>0$, let $\eta=\eta(\varepsilon)$ denote the volume of a ball of radius $\varepsilon / 2$ in a euclidean space of dimension $n(=\operatorname{dim} M)$. Let $B_{p}(r)$ denote the open ball of radius $r$ about $p$ in $M$. We first show that if $\operatorname{vol}\left(\pi B_{q}(\varepsilon / 2)\right)<$ $\eta$ for $q \in M$, then there is $\varphi(\neq 1) \in \Gamma$ such that $d(q, \varphi(q))<\varepsilon$. In fact, if $d(q, \varphi(q)) \geqslant \varepsilon$ for all $\varphi(\neq 1) \in \Gamma$, then $B_{q}(\varepsilon / 2)$ is entirely contained in the Dirichlet region of $\Gamma$ with center $q$. Since the exponential map is distance increasing,

$$
\operatorname{vol}\left(\pi B_{q}(\varepsilon / 2)\right)=\operatorname{vol}\left(B_{q}(\varepsilon / 2)\right) \geqslant \eta .
$$

We choose $t_{0}$ so that

$$
\operatorname{vol}(M / \Gamma)-\operatorname{vol}\left(\pi B_{g(0)}(t-\varepsilon / 2)\right)<\eta \quad \text { for } t \geqslant t_{0}
$$

Since $\pi \circ g$ is a minimizing geodesic, we have

$$
\pi B_{g(0)}(t-\varepsilon / 2) \cap \pi B_{g(t)}(\varepsilon / 2)=\varnothing,
$$

and, for $t \geqslant t_{0}$,

$$
\operatorname{vol}\left(\pi B_{g(t)}(\varepsilon / 2)\right)<\operatorname{vol}(M / \Gamma)-\operatorname{vol}\left(\pi B_{q}(t-\varepsilon / 2)\right)<\eta .
$$

Hence there exists $\varphi(\neq 1) \in \Gamma$ with $d(g(t), \varphi(g(t)))<\varepsilon$.

5. Proof of Theorem. Suppose that $-a \leqslant K<0$ for $a>0$. Let $\Gamma$ be a properly discontinuous group of isometries of $M$ without elliptic elements. Suppose that $M / \Gamma$ is of finite volume and is noncompact. Then there is a minimizing geodesic ray $\pi \circ g$ in $M / \Gamma$ which goes to $\infty$. By Proposition 4 , for any natural number $m$, we can find an isometry $\varphi_{m} \in \Gamma$ with $\min _{p \in M} d_{\varphi_{m}}(p)$ $<1 / m$.

If all $\varphi_{m}$ were hyperbolic, we could pick a subsequence $\left\{\varphi_{m^{\prime}}\right\}$ of $\left\{\varphi_{m}\right\}$ such that $\left\{\Delta\left(\varphi_{m}\right)\right\}$ are not conjugate to each other. Then

$$
C\left(\Delta\left(\varphi_{m^{\prime}}\right)\right) \cap C\left(\Delta\left(\varphi_{n^{\prime}}\right)\right)=\varnothing \quad \text { for } m^{\prime} \neq n^{\prime},
$$

and $\operatorname{vol}\left(C\left(\Delta\left(\varphi_{m^{\prime}}\right)\right)\right) \geqslant c(=\mathrm{a}$ constant $)$, which is a contradiction.

The converse is well known.

\section{REFERENCES}

1. R. L. Bishop and B. O'Neill, Manifolds of negative curvature, Trans. Amer. Math. Soc. 145 (1969), 1-49.

2. P. Eberlein and B. O'Neill, Visibility manifolds, Pacific J. Math. 46 (1973), 45-109.

3. D. Gromall and J. A. Wolf, Some relations between the metric structure and the algebraic structure of the fundamental groups in manifolds of nonpositive curvature, Bull. Amer. Math. Soc. 77 (1971), 545-552.

4. M. Gromov, Manifolds of negative curvature, preprint.

5. __, Almost flat manifolds, preprint. 
6. E. Heintze, Mannigfaltigkeiten negativer Krümmung, preprint, 1976.

7. B. Lawson and S. T. Yau, Compact manifolds of nompasitive curoature, J. Differential Geometry 7 (1972), 211-228.

8. W. P. Thurston, The geometry and topology of 3-manifolds, preprint, 1978.

9. S. T. Yau, On the fundamental group of compact manifolds of non-pasitive curvature, Ann. of Math. 93 (1971), 579-585.

School of Mathematics, Institute for Advanced Study, Princeton, New Jersey 08540

Current address: Department of Mathematics, National Tsing Hua University, Hsinchu, Taiwan 300, Republic of China 